\title{
The bacterial interlocked process ONtology (BiPON): a systemic multi-scale unified representation of biological processes in prokaryotes
}

Vincent J. Henry ${ }^{1,2+}$, Anne Goelzer ${ }^{2^{*}+} \mathbb{D}$, Arnaud Ferré1, Stephan Fischer ${ }^{2}$, Marc Dinh², Valentin Loux², Christine Froidevaux ${ }^{1}$ and Vincent Fromion ${ }^{2}$

\begin{abstract}
Background: High-throughput technologies produce huge amounts of heterogeneous biological data at all cellular levels. Structuring these data together with biological knowledge is a critical issue in biology and requires integrative tools and methods such as bio-ontologies to extract and share valuable information. In parallel, the development of recent whole-cell models using a systemic cell description opened alternatives for data integration. Integrating a systemic cell description within a bio-ontology would help to progress in whole-cell data integration and modeling synergistically.

Results: We present BiPON, an ontology integrating a multi-scale systemic representation of bacterial cellular processes. BiPON consists in of two sub-ontologies, bioBiPON and modelBiPON. bioBiPON organizes the systemic description of biological information while modelBiPON describes the mathematical models (including parameters) associated with biological processes. bioBiPON and modelBiPON are related using bridge rules on classes during automatic reasoning. Biological processes are thus automatically related to mathematical models. 37\% of BiPON classes stem from different well-established bio-ontologies, while the others have been manually defined and curated. Currently, BiPON integrates the main processes involved in bacterial gene expression processes.

Conclusions: BiPON is a proof of concept of the way to combine formally systems biology and bio-ontology. The knowledge formalization is highly flexible and generic. Most of the known cellular processes, new participants or new mathematical models could be inserted in BiPON. Altogether, BiPON opens up promising perspectives for knowledge integration and sharing and can be used by biologists, systems and computational biologists, and the emerging community of whole-cell modeling.
\end{abstract}

Keywords: Systems biology, Multi-scale systemic description, Prokaryotic biological processes, Mathematical models, Biological ontology

\footnotetext{
* Correspondence: Anne.Goelzer@inra.fr

${ }^{\dagger}$ Equal contributors

${ }^{2}$ INRA, UR1404, MalAGE, Université Paris-Saclay, Jouy-en-Josas, France

Full list of author information is available at the end of the article
} 


\section{Background}

Systems biology emerged as a promising framework to integrate the whole-cell for different model-organisms [1-3]. However, current cell representations usually refer to specific model organisms, which limits in practice the transfer of whole-cell models to non-model organisms. In contrast, bio-ontologies are a suitable framework for systematically describing biological objects and thus facilitating knowledge transfer among organisms [4, 5]. In this paper, we address the following question: how to combine systems biology and bio-ontology?

Systems biology has its roots in engineering science and conceptualizes the cell as a system composed of interacting sub-systems [1, 6-11]. In this context, cellular processes are typically described as biological subsystems whose inputs (e.g. metabolites, proteins, or sequences, etc.) are converted into outputs by dedicated molecular machines. The molecular machines are usually composed of proteins, consume energy and chemical building blocks, and display a characteristic of operation. This operation can be static or dynamic, deterministic or/and stochastic and is generally described by a formal mathematical model having inputs, outputs and model parameters. For example, a mathematical model can be a nonlinear function or a set of ordinary differential equations. The systemic representation of cells is an efficient framework to interrelate all cellular entities (metabolites, proteins, cellular processes, sequences, etc.), together with their physical or biochemical properties (e.g. kinetic parameters, etc.) $[1,2]$. System biologists thus need now an adequate format of systemic description of the whole cell to transfer and share their models. Existing standardized formats for file exchange are adequate to exchange mathematical models for specific cell processes [12, 13], but remain limited to describe a whole-cell model, i.e. a systemic multi-scale representation of interacting complex subsystems.

Bio-ontologies have been developed to formalize and integrate different pieces of biological knowledge [4]. The well-established Gene Ontology (GO) integrates the molecular functions of gene products (GO-MF) with cellular components (GO-CC) and biological processes (GO-BP) [14]. The combined sub-ontologies are commonly used to annotate and characterize gene products $[5,15]$, but there are also other useful bio-ontologies. The Ontology of Microbial Phenotypes links the phenotypes of bacteria to cellular processes [16]. The Ontology of Genes and Genomes provides a list of genes from different organisms including prokaryotes [17], while the Sequence Ontology (SO) provides a detailed description of polymers and polymer sequence patterns [18]. At another level, the Pathway Ontology (PW) provides a classification of metabolic, signaling and altered eukaryotic pathways [19]. Independently, ChEBI (Chemical Entities of Biological Interest) acts as a reference for the classification of general chemicals according to their chemical structures and modifications [20]. The Systems Biology Ontology (SBO) provides a controlled vocabulary for kinetic parameters and mathematical models of biological processes [21]. Taken together, the existing bio-ontologies cover the concepts necessary to the systemic representation of cells, i.e., biological processes, molecules and mathematical models of biological processes. However, the systemic representation of the whole cell cannot be handled without the addition of further logical relations between existing ontologies.

In this paper, we demonstrates that a systemic multiscale representation of biological processes, the typical perspective of systems biology, can be formally described as an ontology, and how this ontology can be built based on existing sparse bio-ontologies. As a proof of concept, we developed the Bacterial interlocked Process ONtology (BiPON) and showed that a) heterogeneous biological processes can be described with the systemic representation and b) be linked automatically to mathematical models, and that c) information about these processes can be enriched by automatic reasoning. As a use case, we focus on bacterial gene expression processes, which are well established and representative of known biological processes. They cover, among many other things, combination of polymers, sequence patterns, single molecules or complexes within biological processes, as well as cyclic or branched-point processes. We demonstrated on the use case how a systemic representation of living cells can be formally described and integrated into an ontological model, and what benefits ensue from automatic reasoning on this ontology.

\section{Methods}

\section{Description of biological processes, corpus building and entity tagging}

In the absence of an exhaustive controlled vocabulary in systems biology, we use hereafter the notion of a "biological process", which comprises the notions of (a) "biological reaction" and "biochemical reaction" as in KEGG (Kyoto Encyclopedia of Genes and Genomes [22]) Reactions database, (b) "biological phenomenon", "biological pathway" and "biochemical pathway" as in PW or KEGG Pathway database, and finally (c) "biological process" as in GO-BP. Moreover, we use the notion of a "chemical entity" to denote any type of biological compound, including metabolites, proteins, protein complexes, polymers, to cite a few.

To develop a dedicated systemic representation for each biological process involved in the bacterial gene expression, we applied the standard state-of-art approach of system engineering. The approach involves two main tasks. (A) We first gathered up-to-date available biological information about the biological process. (B) We 
then converted the biological information into a systemic representation using boxes, arrows, inputs and outputs, and a mathematical model. We describe and apply below the approach (A) and (B) on a specific example (the formation of the $30 \mathrm{~S}$ initiation complex) for illustrative purposes. Note that the approach is generic and can be applied on any biological process.

(A)We collected up-to-date knowledge about the biological processes from scientific literature (books, peer-reviewed original articles, and reviews; see Additional file 1 for a list of references). We primarily focused on figures since they facilitate the conversion from biological knowledge to the systemic representation in the task (B) (as illustrated in Fig. 1).
Elementary steps composing a biological process are usually found in research articles while books or review articles provide global descriptions of processes. In a few cases, we used figures from didactic web sites and we checked the biological information using original research papers systematically.

(B)We then converted the selected figure into a systemic representation. Despite the heterogeneity of sources, several common features were identified from these schemas (as illustrated Fig. 1): title (t), arrows (a) and shapes (s) with legend or label (1).

\section{Tagging entities of interest}

Given a graphical representation of any biological process with sub-processes (Fig. 1):

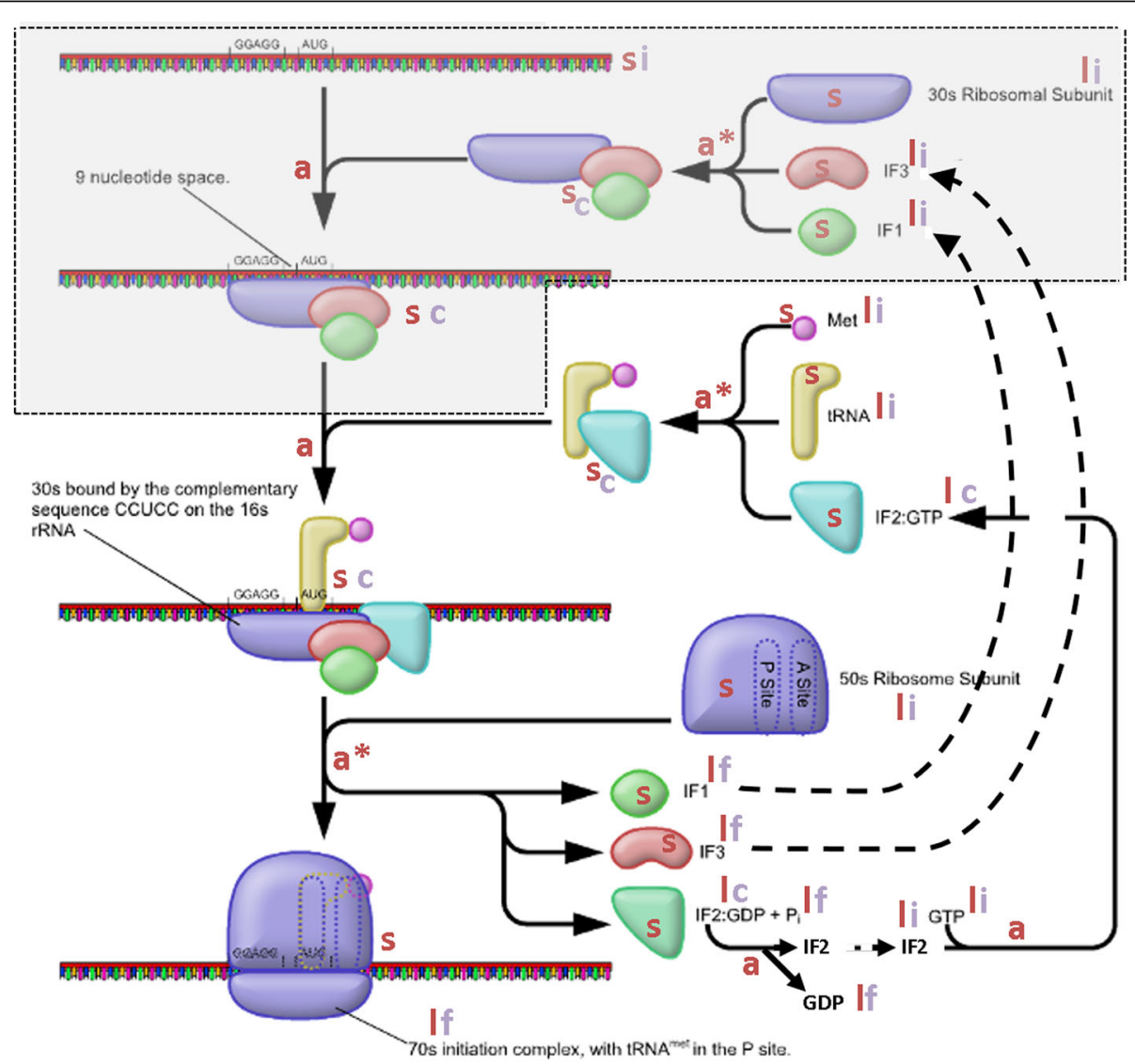

$\mathrm{t}$ The process of initiation of translation in prokaryotes.

Adapted from "The process of initiation of translation in prokaryotes." By Richard Wheeler (Zephyris) 2005; Wikimedia Commons, the free media repository

Fig. 1 From biological knowledge to systemic description: Identification of the entities of interest for the process of initiation of translation in prokaryotes on a representative figure extracted from Wikimedia. Entities for systemic model design are marked by the following symbols: title (t); arrows divided in bifid at origin or head (a) or divided in more than two parts $\left(\mathbf{a}^{*}\right)$ or closing cycle (dotted); shapes (s) with legend or label (I). Depending on their relative position regarding arrows (origins or heads), three types of biological entities (BioE) are identified (i, $\mathbf{f}, \mathbf{c})$ : an unframed BioE at arrow-origins (i) represents an initial reactant of a process (input); an unframed BioE at arrow-heads (f) represents a final product of a process (output); a framed BioE (c) represents a product (output) of a process that is the reactant (input) of the next process, and thus that consumed BioE 
- The title (t) defines the name of the main biological process that embeds the succession of all identified individual processes.

- The arrows are identified as sub-reactions that correspond to the individual processes. Three types of arrow are distinguished on Fig. 1: linear (dotted), bifid at origin or head (a) and divided in more than two parts $\left(\mathbf{a}^{*}\right)$.

- The shapes (s) are identified as the chemical entities (BioE) that participate in a biological process and are related to legends or labels (1). Depending on their relative position regarding arrows (origins or heads), three types of BioE are identified (i, f, c): an unframed BioE at arroworigins (i) represents an initial reactant of a process (input), called iBioE; an unframed BioE at arrow-heads (f) represents a final product of a process (output), called fBioE; a framed BioE (c) represents a product of a process (output) which is the reactant of the next process (input), called cBioE (for consumed BioE).

Note that

- Arrows that correspond to BioE recycling within a process are not considered (as illustrated by the dotted arrow in Fig. 1).

- Any BioE may be an initial reactant and/or a product of several distinct processes.

\section{Biological processes as interlocked systems}

After identifying the entities necessary in the biological process, we organized them as a main system composed of different interlocked sub-systems of lower granularity, as follows.

An elementary process is formally defined by its participants, i.e. the input(s) and output(s). The standard systemic representation of an elementary process corresponds to a box framed by its input(s)/output(s) (see Fig. 2a). In this graphical representation, inputs are placed on the left of the box at the tail of the incoming arrows, while outputs are placed on the right of the box at the head of the outgoing arrows (Fig. 2a). In our biological context, an elementary process corresponds to a biological reaction and the inputs are the BioEs required for the production of the BioEs that served as outputs.

\section{Multi-scale representation of processes}

In a multi-scale representation, the same process is represented at different levels of granularity (Fig. 2b). On the top level of granularity, there is a unique aggregated process that leads to output(s) (B1 in a dark gray box). An aggregated process can be formally defined either by its input(s)/output(s), like an elementary process, or by the composition of successive sub-processes. On the bottom level of granularity, there is a succession of elementary processes that lead to the same output(s) as those produced by other levels (B3 in white boxes). Via decomposition and aggregation of processes, we can

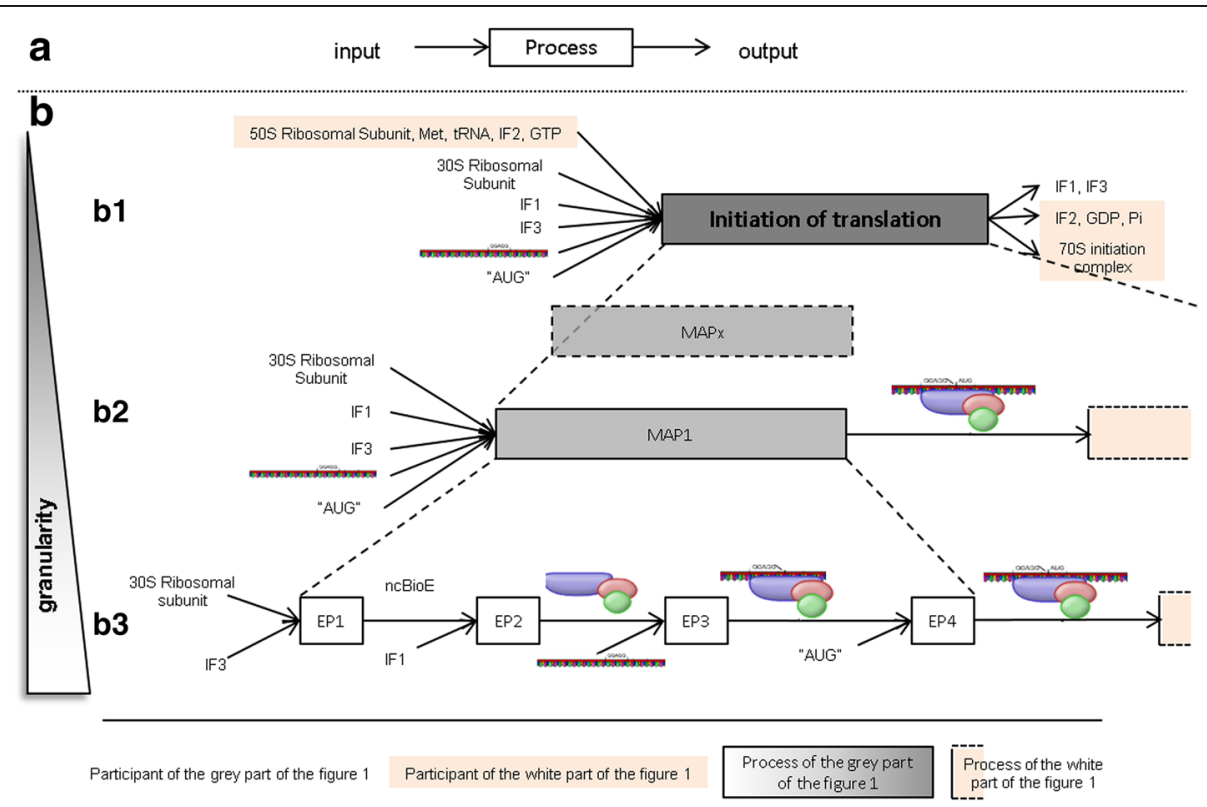

Fig. 2 Systemic decomposition of biological processes. a Input/output representation of a generic process. b Decomposition of a biological process (e.g. initiation of translation) (level B1) into sub-processes of higher granularity, down to the decomposition into elementary processes (level B3). Abbreviations are EP for Elementary Process, MAP for Middle Aggregated Process, ncBioE for next consumed biological entity 
navigate between the different levels of granularity (represented by a gray scale on Fig. 2b).

\section{Systemic model of the main process (level B1 on fig. 2b)}

The fully aggregated process (at the lowest granularity level) is the main process having iBioEs as inputs and fBioEs as outputs. In the graphics, it is represented by a box and labeled according to the name of the global reaction. The box is framed by BioEs, one iBioE per input of the main process on the left, and one fBioE per output of the main process, on the right.

\section{Systemic model of elementary processes (level B3 on fig. 2b)}

An elementary process is a sub-reaction of an aggregated process (arrows in Fig. 1), having typically one or two inputs and one or two outputs. In Fig. 1, such a reaction usually concerns bifid arrows (case a). In the case of arrows divided into more than two parts (case $\mathbf{a}^{*}$ on Fig. 1), and thus implicating at least three inputs or outputs, the process is further split into a sequence of elementary processes through the addition of new consumed BioEs (ncBioE), using additional literature information when available. Two successive elementary processes which share a common participant, i.e. an output of the first elementary process is an input of the second one (cBioE). Elementary processes follow each other until all outputs of the main process are produced (B1 level). Note that cBioEs and ncBioEs never appear as participants in the main process (the fully aggregated one).

\section{Systemic model of intermediate processes (level B2 on fig. 2b)}

Intermediate processes provide intermediary levels of granularity between the main process and the elementary processes. In the graphical representation, an intermediate process consists of a box that merge boxes of elementary processes. Intermediate processes define sub-processes of specific biological interest. They are built by aggregation of successive elementary processes, following biological considerations, e.g. about the presence of irreversible reactions, the relevance of an intermediate process and of the special nature of a BioE, or the capability to experimentally detect or quantify a specific BioE.

\section{Mathematical models of biological systems}

In systems biology, the community has investigated and developed numerous mathematical models [23, 24] enabling the description, analysis, and simulation of biological processes. Mathematical models can be very different in nature (static, dynamical, stochastic, etc.) and depend on various parameters and variables. One biological process can be described with several mathematical models. For instance, protein translation can be modeled by deterministic [25] or by stochastic models
[26]. Conversely, several biological processes can have the same type of mathematical model, such as the Michaelis-Menten equation for the kinetics of different enzymes. In the bio-ontology BiPON, we formalize the relation between biological processes and their mathematical description(s).

\section{BiPON design}

BiPON is a bio-ontology that is composed of two subontologies: bioBiPON and modelBiPON. bioBiPON organizes the systemic description of biological information, while modelBiPON describes the mathematical models associated with biological processes. In the following, a class that has no sub-class for the property is_a is called a leaf-class. BiPON has been designed using the software editor Protégé 5 and the Description Logic Manchester syntax [27].

\section{bioBiPON ontological model Main classes}

BioBiPON contains four main classes, which corresponds to the main structure of major bio-ontologies: Biological process (GO:0008150), Chemical entity (CHEBI:24,431), Sequence feature (SO:0000110) and Cellular component (GO:0005575).

The classes Biological process and Cellular component include a selection of GO classes, while the Chemical entity class includes a selection of ChEBI classes for small molecules, of SO classes for gene products (e.g. primary transcript), and terms of the KEGG database orthology (KO) for proteins [22]. The Sequence feature class includes a selection of SO classes for sequence patterns. Finally, classes which were not present in existing bio-ontologies were created manually.

The Biological process class contains as subclasses the biological processes and sub-processes (irrespective of their granularity level). The Chemical entity class contains as subclasses the participants (BioE) of a biological process, e.g. molecules, proteins, molecular complexes, polymers, etc. The Sequence feature class contains as subclasses any sequence patterns carried by molecules. Polymers such as DNA and RNA (which belong to the class Chemical entity) act as template (have a matrix role): they carried different sequences patterns (e.g. promoter sequences, transcription factor binding site, ribosome binding site, pausing site for ribosomes, etc.). Some of these polymers can participate in several processes. For instance, the same mRNA can be an input of the translation process and of the mRNA degradation process. However, the molecular complexes or proteins involved in these distinct biological processes recognize the sequence patterns. For instance, in Figs. 1 and 2, the specific mRNA sequence patterns named "GGAGG" and "AUG" are involved in two successive elementary processes. Effectively, the 
inputs of these processes are thus the sequence patterns, and not the whole mRNA itself. When a process is decomposed as in the previous example, we choose to use the sequence patterns of polymers as process participants instead of the molecules themselves. In addition, sequence patterns and molecular complexes have to interact and thus have to share a common localization on chromosomes or mRNAs. We defined the Cellular component class, which contains as subclasses the parts of cells in which molecules can be localized, and the polymers that carried sequence patterns or bounded chemical entities. In the case of bacteria (a cell without organelle), Cellular component class contains the cytosol and polymers such as chromosome or mRNA.

Class hierarchy and subclass property Inside the four main classes, subclasses are organized according to the is_a relation to get a Directed Acyclic Graph (DAG) structured model. Unlike in a tree, a class can not only have several subclasses but also be a subclass of several classes (multiple inheritance). The hierarchy of the classes that were imported from GO, ChEBI and SO is kept within the DAG model. Processes, chemical entities and patterns are placed as leaves of the bioBiPON DAG model.
Importation and interoperability For all classes and properties that were imported from other bio-ontologies (e.g. translation initiation; see Fig. 3), we kept the original references, such as the Internationalized Resource Identifier (IRI) and Identifier (id) in bioBiPON, to ensure interoperability. In BiPON, the $\mathrm{SO}$ classes of gene products are now considered as subclasses of the Chemical entity class instead of the Sequence feature class. Due to this semantic change, we considered these SO classes as new classes: we gave a new IRI and kept the original one with the hasDbXref annotation (e.g. hasDbXref SO_0000185). When a class refers to a term in an existing database (such as $\mathrm{KO}$ ), the original id is also kept with the hasDbXref annotation (e.g. prokaryote translation initiation factor IF-3: hasDbXref K02520; see Fig. 3).

Labeling For any imported class, the original label is still used in bioBiPON. For any newly created class, we have manually defined a label that was the most representative of the biological process, molecule or sequence represented by the class. The final label can be (a) a term commonly used in the biological schemes of peer-reviewed articles that we considered, or else (b) a Wikipedia term and, otherwise, (c) a term that we chose by taking into account length, completeness and non-ambiguity criteria.

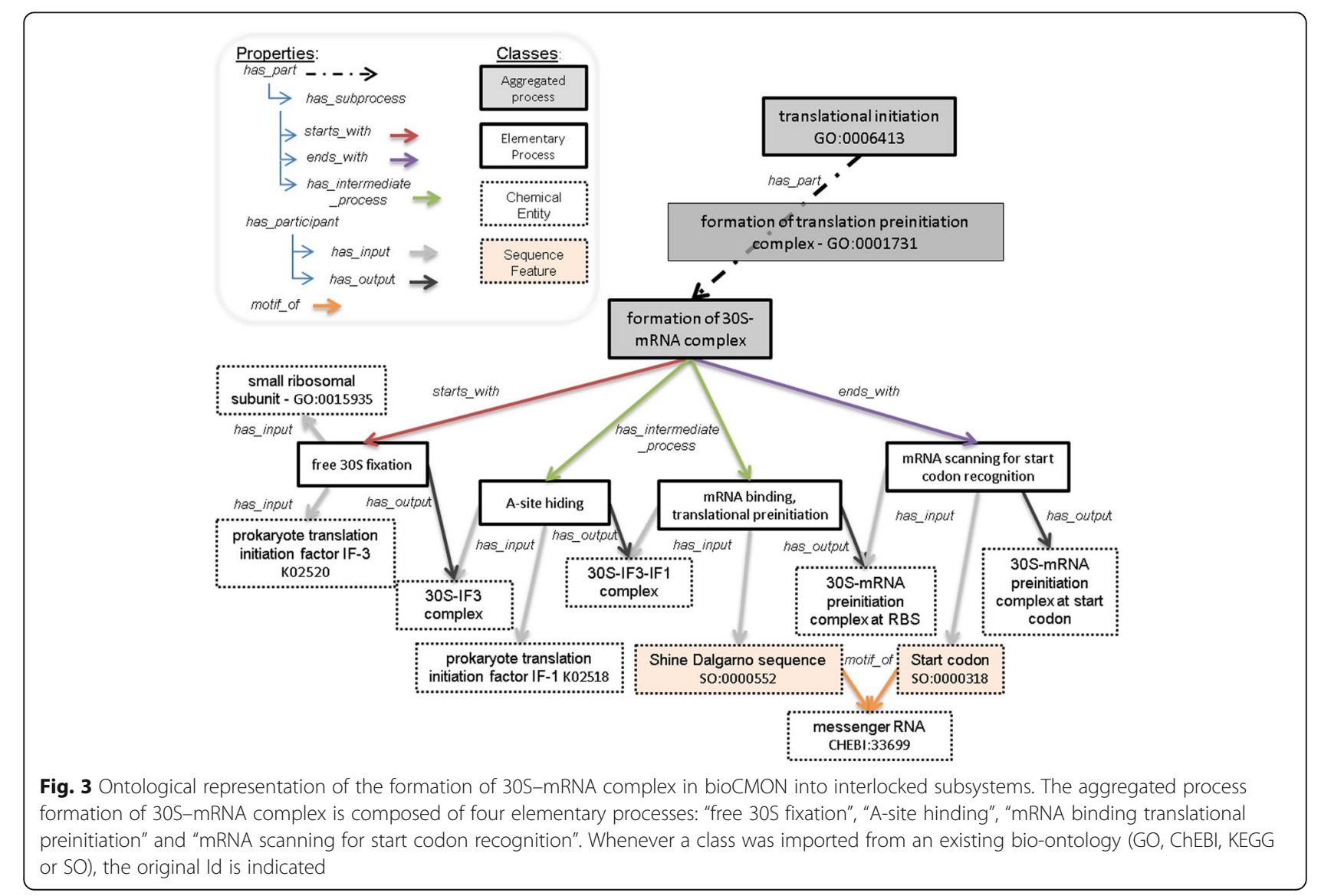




\section{Main properties}

Properties were partly imported from the Relation Ontology (RO) [28] and partly created manually. Two main properties, has_participant (RO_0000057; (INVERSE OF participates_in RO_0000056)) and has_part (BFO_0000051) were used to formalize elementary or aggregated processes, respectively. The has_participant property includes the sub-properties has_input (RO_0002233), has_output (RO_0002234), and has_catalyst. In the ontological model, they are represented by arrows between the biological processes and the BioEs (see Fig. 3). These properties are used to formalize relations between elementary processes. The has_part property is transitive and is further specialized into two intransitive sub-properties called cyclication_of and has_subprocess. The has_subprocess is further specialized into starts_with, ends_with, has_intermediate_process and has_fork_process disjoint sub-properties that can be used to formalize aggregated processes. The has_part property enables the decomposition of an aggregated process along the granularity levels down to elementary processes, while the has_subprocess property manages the relation between two successive granularity levels. starts_with, ends_with, has_intermediate_process and has_fork_process participate in the management of successive processes that are part of a process of the same granularity level. The properties starts_with and ends_with define which sub-process starts and ends the aggregated process respectively. We further define the property has_fork_process in the case of several sub-processes start an aggregated process. The properties has_intermediate_process define the sub-processes that occur between the starting and the ending sub-processes.

The located_in property is used to define the localization of Chemical entity class inside the cell.

As mentioned above, the Chemical entity and Sequence feature classes are in relation through the is_motif_of, binds_to and has_template properties. The transitive property is_motif_of localizes the sequence patterns in a larger one and finally in a polymer. The binds_to property (a located_in sub-property) defines the sequence where a Chemical entity binds a polymer. The has_template property points out a sequence that affects the recruitment of a specific Chemical entity.

Formal definition of biological processes We used the Protégé editor, which is based on Description Logics, to formalize the classes. We distinguished two kinds of classes, namely primitive classes, which are described by necessary conditions (e.g. subclass of other classes), and complex classes, which are defined by equivalence using both necessary and sufficient conditions. Thus, the formal definition of classes follows templates that may combine universal (ONLY) and existential (SOME) restrictions [27]. The structure of bioBiPON is displayed on Fig. 4.

Elementary process class is related to chemical entity or sequence features classes via has_participant subproperties by the following general class axiom:

elementary_process $\equiv$ has_input SOME chemical entity AND has_output SOME chemical entity AND has_input ONLY (chemical entity OR sequence feature) AND has_output ONLY (chemical entity OR sequence feature).

In the previous definition, Chemical entity is a primitive class (defined as a subclass of bioBiPON), while elementary_process is a class defined by equivalence using two kinds of restrictions. Any subclass of elementary_process must have at least one Chemical entity subclass as an input and as an output. Moreover, the inputs and the outputs of a subclass of elementary_process must be either subclasses of Chemical entity, either subclasses of Sequence feature.

For instance, the elementary process subclass Free $30 S$ fixation in Fig. 3 is defined as follows:

Free 30S fixation $\equiv$ has_input SOME IF3 AND has_input SOME 30S AND has_output SOME 30S-IF3 complex AND has_input ONLY (IF3 OR 30S) AND has_output ONLY 30S IF3 complex.

In this definition of the class Free $30 S$ fixation, we specialized the type of chemical entity that at least one input (output) must satisfy. For instance, one input has to belong to the class IF3, a sub-class of chemical entity.

Aggregated process class are related to cellular process class via has_part sub-properties according to the following general class axiom:

aggregated_process $\equiv$ has_subprocess SOME cellular process AND has_subprocess ONLY cellular process.

For instance, the aggregated process subclass Formation of 30S-mRNA complex in Fig. 3 is defined as follows:

Formation of 30S-mRNA complex $\equiv$ starts_with SOME free $30 \mathrm{~S}$ fixation AND has_intermediate_process SOME A site hiding AND has_intermediate_process SOME mRNA binding, translation preinitiation AND ends_with SOME mRNA scanning for start codon recognition AND has_subprocess ONLY (free 30S fixation OR A site hiding OR mRNA binding, translation preinitiation OR mRNA scanning for start codon recognition).

Fig. 3 illustrates the ontological representation of the formation of 30S-mRNA complex into aggregated and elementary processes using the classes and properties of bioBiPON.

\section{modelBiPON ontological model}

The ontological model called modelBiPON aims at relating generic biological processes to their mathematical models including parameters. Knowledge about mathematical models was gathered from two sources. A first flat source of knowledge was provided by systems biology 


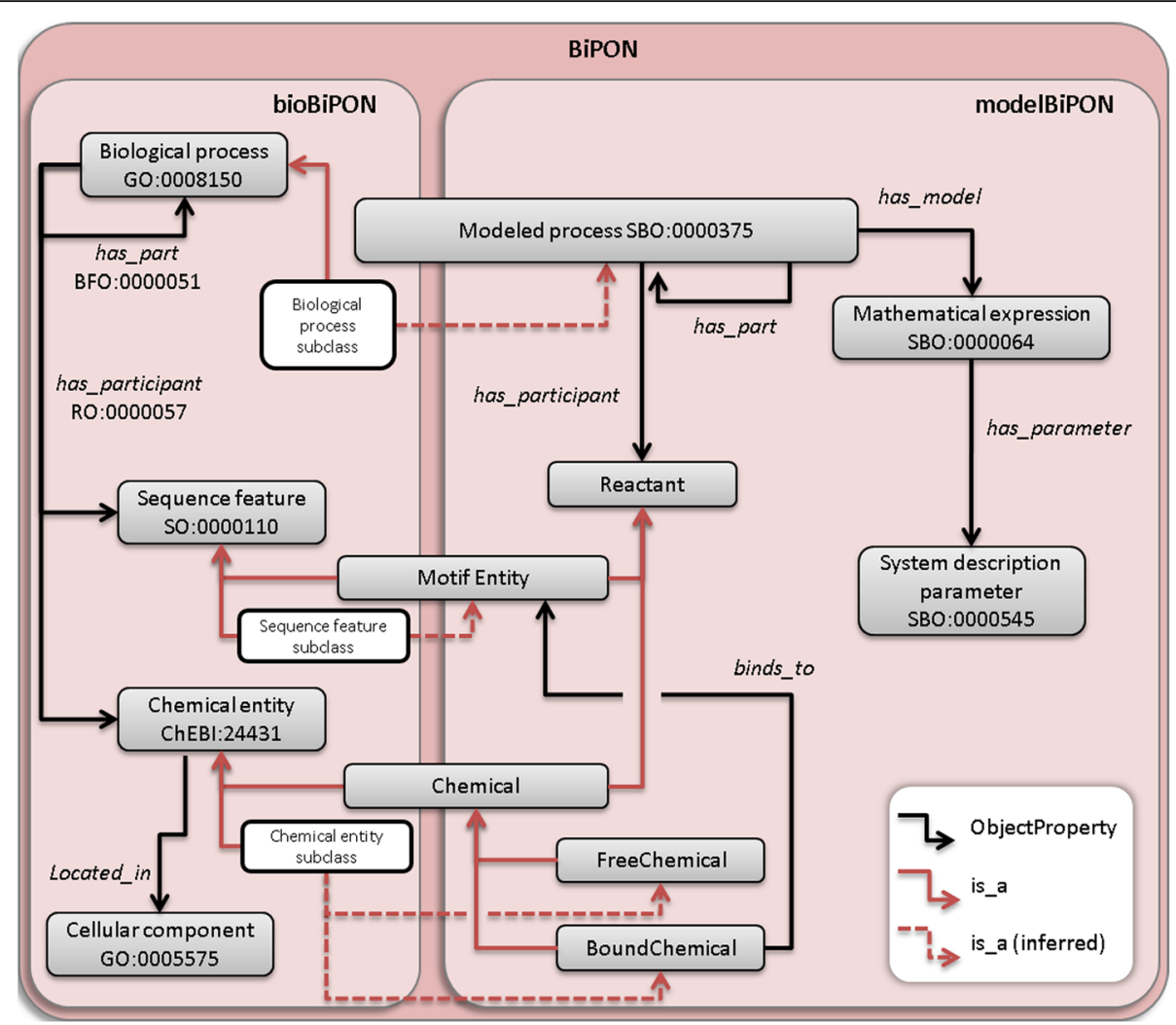

Fig. 4 BiPON structure: focus on automatic reasoning on bioBiPON and modelBiPON. Dotted red arrows represent the inferred relation between classes of bioBiPON and modelBiPON using automatic reasoning, while straight red arrows stand for the standard is_a property. Abbreviations are GO: Gene Ontology; SO: Sequence Ontology; ChEBI: Chemical Entities of Biological Interest; SBO: System Biology Ontology; BFO: Basic Formal Ontology; RO: Relation Ontology

specialists who established a list of generic, useful and wellestablished models of biological processes. The second source of knowledge was a selection of ontology classes that were directly imported from SBO, more specifically from the mathematical expression (SBO:0000064) and the system description parameters (SBO:0000545) classes. These classes and subclasses include fairly enough pieces of knowledge for laws and parameters, respectively.

\section{Main classes}

We defined four main classes: Modeled process, Reactant, Mathematical expression and System description parameters (Fig. 4).

The Modeled process class corresponds to the process class of SBO (SBO:0000375) and contains, as subclasses, specific biological processes of bioBiPON for which it exists a mathematical model. The Reactant class is an abstract representation of the inputs and outputs of a Modeled process. Reactant is specialized into two disjoint subclasses, Motif Entity and Chemical, corresponding to the subclasses of Sequence feature and Chemical entity of bioBiPON that are inputs/outputs of a Biological process (see Fig. 4). The Chemical class is further specialized into the disjoint subclasses Free Chemical and Bound Chemical. Subclasses of Free Chemical represent the chemical entities that are freely available to interact with any other chemical entities in the cytoplasm. The subclasses of Bound Chemicals represent molecular complexes composed of one or several chemical entities that are bound specifically to a sequence pattern. The Modeled process and Reactant classes are abstract representations and are therefore at the top level of the modelBiPON ontology.

Mathematical expression and System description parameters include subclasses from $\mathrm{SBO}$ and subclasses that were defined according to the mathematical models. For the classes that were imported from SBO, we carefully kept the IRIs and the Ids to ensure interoperability between ontologies.

\section{Main properties}

In modelBiPON, the sub-properties of has_participant in bioBiPON were used to relate the classes of Modeled 
process and Reactant while the sub-properties of has_part managed the decomposition of Modeled process. Two new types of properties were defined (Fig. 4): has_model and has_parameter. The has_model property links the Modeled process and Mathematical expression classes while the has_parameters property links the Mathematical expression and System description parameter classes (Fig. 4).

\section{Formal definition of modeled processes}

The Modeled process subclasses are defined by the specificity of their participants (belonging to the class Reactant) or by the nature of their discriminating sub-processes. For instance, the most common process belonging to Modeled process is elementary chemical process. By definition, an elementary chemical process has exclusively participants in the Chemical class:

elementary chemical process $\equiv$ elementary process AND has_input ONLY Chemical AND has_output ONLY Chemical.

In the same way, a Sequence binding process corresponds to the elementary process of binding a FreeChemical to a Motif Entity and leads to the formation of a BoundChemical. This process is formalized as follows:

sequence binding process $\equiv$ elementary process AND has_input SOME Motif entity AND has_input SOME FreeChemical AND has_output SOME BoundChemical AND has_input ONLY (Motif entity OR FreeChemical) AND has_output ONLY BoundChemical.

Aggregated processes that are included in Modeled process might be defined by the nature of their discriminating sub-processes such as Matrix dependent process and Polymer production process:

matrix dependent process $\equiv$ aggregated process AND has_part SOME Sequence binding process.

polymer production process $\equiv$ matrix dependent process AND has_part SOME Release process.

In the previous formal definition, Release process is also a subclass of Modeled process.

Finally, a Modeled process can be refined using the biological property of its participants. For example, the transcription process and translation process are defined in modelBiPON as follows:

Transcription process $\equiv$ native polymer production process AND has_output SOME primary_transcript.

Translation process $\equiv$ native polymer production process AND has_output SOME pre-process polypeptide.

\section{BiPON consistency with GO, ChEBI, SO and SBO}

To evaluate the logical consistency of BiPON with respect to $\mathrm{GO}, \mathrm{ChEBI}, \mathrm{SO}$ and $\mathrm{SBO}$, we imported the whole set of classes of each ontology into BiPON. Then, we ensured logical consistency using the HermiT 1.3.8 reasoner within the Protégé editor [29].

\section{Results}

\section{Reasoning on bioBiPON using SWRL rules}

Since the BiPON ontology is described using DL syntax, automatic reasoning can be performed within the DL SROIQ framework [29]. However, reasoning on classes alone has its limitations, especially when negations or properties intersection need to be handled [30]. This difficulty can be bypassed by instantiation of classes. We first instantiated leaf-classes with a unique and distinct individual indiv: i_NameOfTheClass, s_NameOfTheClass, and $p_{-}$NameOfTheClass for the Chemical entity, Sequence feature and Biological process leaf-classes respectively. Leaf-classes were restricted to these singletons. We assume that the unique individual indiv is considered as the typical member of its class. Other individuals can be instances of that class, but they will all be inferred to be the same as the typical individual indiv, since each singleton leaf-class is defined as equivalent to \{indiv\}. After the instanciation, we designed rules in Semantic Web Rule Language (SWRL), as supported in Protégé, to formalize additional constraints between classes and properties [30].

\section{Automatic input/output building of aggregated processes}

In the rest of the paper, we call "sub-process of a process $p$ "any process that is related to $p$ by a has_subprocess property.

Elementary processes are manually defined by specifying the classes of their inputs and outputs using has_input and has output properties, while aggregated processes are manually defined by the composition of their subprocesses (see Fig. 5a). The naive composition of has_participant (has_input or has_output) and has_subprocess sub-properties would result in the list of all inputs/ outputs of elementary processes. However, intermediate macromolecules that are produced and consumed by two successive elementary processes should be removed from the inputs/outputs of aggregated processes (see Fig. 5b).

To overcome this difficulty, we built the inputs/outputs of aggregated processes by automatic reasoning using SWRL rules in two steps.

Step1: We identified a first set of input(s) and output(s) of the aggregated process: (a) the input(s) of all subprocesses that start the aggregated process and (b) the output(s) of the sub-process that ends the aggregated process. In formal language, we have:

Let $\mathrm{p}$ _agg be an aggregated process. IF pagg starts with p_sub and p_sub has the molecule chem_i as an input, THEN p_agg has the molecule chem_i as an input. And IF pagg has as fork process p_sub and p_sub has the molecule chem_i as an input, THEN p_agg has the molecule chem_i as an input. And IF pagg ends with p_sub and p_sub has the molecule 


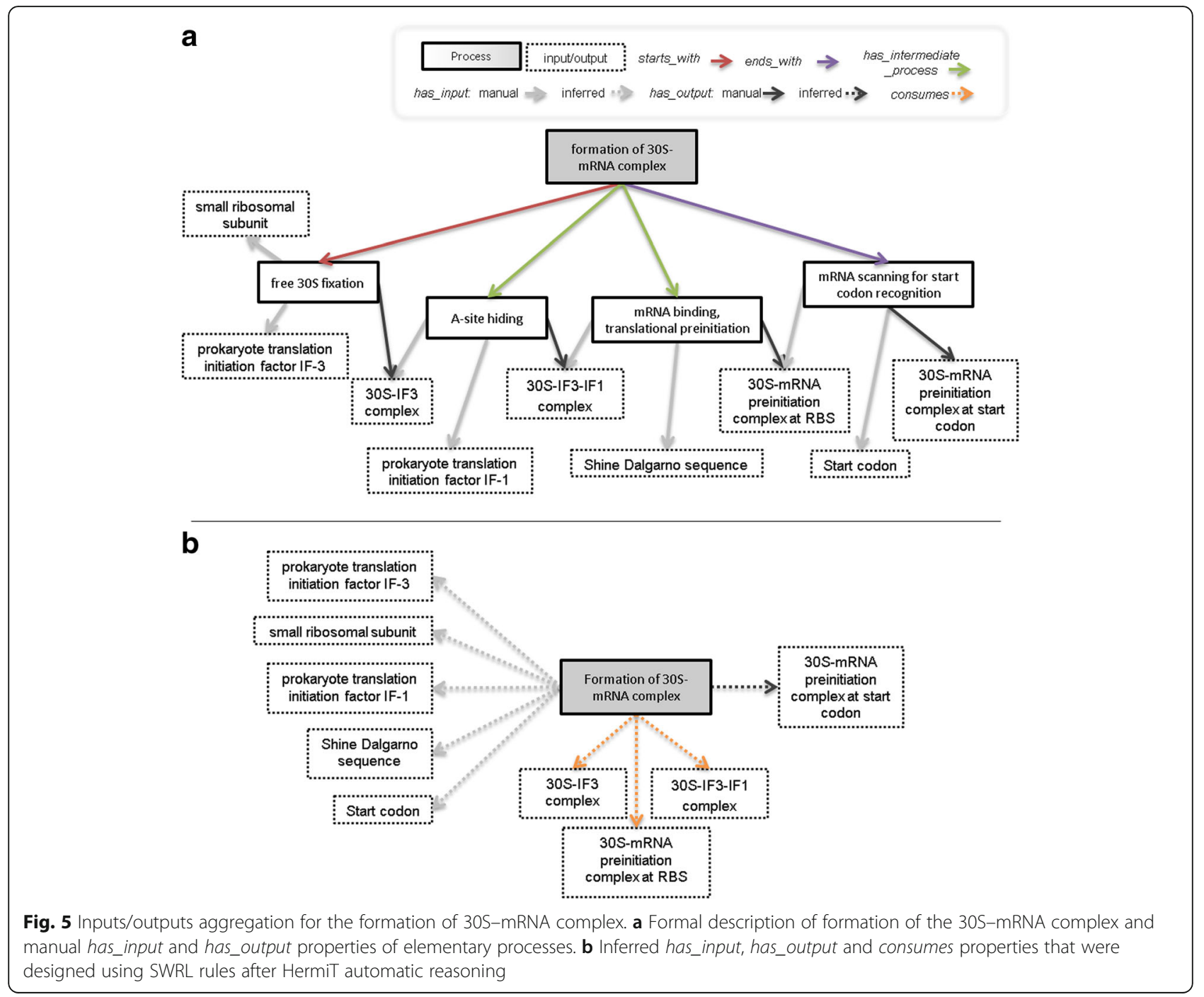

chem_i as an output, THEN p_agg has the molecule chem_i as an output.

Step2: We identified a second set of inputs and outputs of the aggregated process, containing any input or output that is not produced then consumed by two successive intermediate sub-processes. To do so, we first had to determine the successive order of two subprocesses in an aggregated process. We defined the property precedes as follows. Since by definition two successive elementary processes are linked by one intermediate molecule, the process that provides the molecule (as output) precedes the process that consumes the molecule (as input).

Let P_agg be an aggregated process and P_sub1 and p_sub2 be some distinct processes. IF p_sub1 and p_sub2 are sub-processes of p_agg and p_sub1 has the molecule chem_i as an output and p_sub2 has the molecule chem_i as an input, THEN p_sub1 precedes p_sub2.
Then any output of the first sub-process that is not an input of the second sub-process, will be an output of the aggregated process. Conversely, any input of the second sub-process that is not an output of the first sub-process will be an input of the aggregated process.

Let P_agg be an aggregated process that starts with a sub-process, let p_sub1 and p_sub2 be some successive processes, and let chem_i and chem_j be different molecules. IF p_sub1 has the molecule chem_i as an output and $\mathrm{p} \_$sub2 has the molecule chem_ $\mathrm{i}$ as an input and p_sub2 has the molecule chem_j as an input, THEN p_agg has the molecule chem_j as an input.

\section{Automatic identification of consumed participants}

We also identified intermediate molecules that are consumed by an aggregated process, e.g. molecules that are produced and then consumed by two successive elementary processes. 
IF an aggregated process p_agg is composed of subprocesses $\mathrm{p} 1$ and $\mathrm{p} 2$. IF $\mathrm{p}$ _agg starts with process p_starts and ends with process p_ends, IF the macromolecule $\mathrm{m}$ is an output of $\mathrm{p} 1$ and the macromolecule $\mathrm{m}$ is an input of $\mathrm{p} 2$ and $\mathrm{p} 1$ is different from $\mathrm{p} 2$, IF $\mathrm{p} 1$ is different from p_ends, and IF $\mathrm{p} 2$ is different from p_starts, THEN p_agg consumes $\mathrm{m}$.

An example, the aggregation of inputs and outputs of the "formation of 30S-mRNA complex" process is given on Fig. 5. The process is represented twice, before (Fig. 5a) and after (Fig. 5b) aggregation. In Fig. 5b, we highlight the three intermediate molecules (30S-IF3 complex, 30S-IF3-IF1 complex and 30S-mRNA preinitiation complex at RBS) that were identified with the consumes property. These three intermediate molecules do not appear as participants in the aggregated process.

\section{Automatic identification of key participants}

For some elementary processes, an input participant can also appears as an output participant. Such a participant is a reactant that is necessary to the realization of the process, but that is not modified in the process. This participant can then be considered as a key component of the elementary process. An example of such a participant is the enzyme that catalyzes an enzymatic reaction. Identification of the key participants of aggregated processes is obtained after automatic aggregation of inputs and outputs. To identify these key participants by automatic reasoning, we designed the new property has_key_element with an SWRL rule (see Additional file 2 for the formal definition):

IF $m$ is a macromolecule and $p$ has $m$ both as an input and as an output THEN $\mathrm{p}$ has $\mathrm{m}$ as a key element.

The key elements of a process can further be linked to this process by means of the inverse property of has key_element: key_element_of. A macromolecule may be a key element of several different processes, which in turn may be involved in different cellular functions. The property key_element_of enables to find all processes for which a macromolecule appears as a key element. For instance, the "Class I translation release factor" (RF, BiPON_00000361) is key_element_of the processes "ArfA system rescue" (BiPON_00001193) and "bacterial cytoplasmic translational termination" (BiPON_00002268). By rating this statement, we automatically point out the dual functional role of RF in the translation process. In total, we obtained 44 key elements for the processes contain in the bacterial gene expression.

\section{Reasoning on bioBiPON and modelBiPON}

To establish relationships between the sub-ontologies bioBiPON and modelBiPON, we applied automatic inference. We first defined the Reactant class and subclasses of modelBiPON by bridge rules with bioBiPON subclasses. Inference then proceeds in two steps based on the formal definition of modelBiPON (Fig. 4 red dotted arrow): (1) a linking operation between Chemical entity or Sequence feature of bioBiPON and Reactant of modelBiPON, and then (2) a hierarchical classification of Biological process of bioBiPON within Modeled process of modelBiPON according to their model and participant characteristics obtained in step 1 through the is_a property. Modeled process and Reactant classes from modelBiPON are then filled with a set of Biological process and Chemical entity subclasses from bioBiPON, respectively. This consists in an automatic selection of biological processes for which a mathematical model exists (Fig. 4 dotted red arrow).

\section{Definition of bridge rules}

For the first step of automatic inference, bridge rules between the classes of both ontologies must be defined. The Reactant, Chemical, and Motif Entity classes as well as their subclasses in modelBiPON are related, via these rules, to the Chemical entity and Sequence feature classes in bioBiPON. More precisely, the class Reactant is defined as follows:

Reactant $\equiv$ participates_in SOME biological process.

As stated in the Methods section, the Chemical and Motif Entity subclasses of Reactant are disjoint. The Chemical class includes subclasses of Chemical entity, while subclasses of Motif Entity in modelBiPON correspond to subclasses of Sequence feature in bioBiPON:

Chemical $\equiv$ Reactant AND Chemical entity.

Motif Entity $\equiv$ Reactant AND Sequence feature.

Moreover, due to their importance for modeling purposes, we formally defined the Free Chemical and Bound Chemical subclasses as follows:

FreeChemical $\equiv$ Chemical AND located_in ONLY cytosol.

BoundChemical $\equiv$ Chemical AND binds_to SOME Sequence feature.

Since binds_to is a sub-property of located_in, the localization of Bound Chemical corresponds to the one of Motif entity.

\section{modelBiPON filling by automatic reasoning}

The class hierarchy of the Reactant, Chemical, and Motif Entity classes was inferred by automatic reasoning. Once initial inputs and outputs of a Biological process class in bioBiPON are related to the abstract class Reactant of modelBiPON, the Modeled process class hierarchy is performed by automatic reasoning. At last, the most specialized subclasses for the is a relation of the Reactant and Modeled process classes in modelBiPON are the leafclasses of bioBiPON. Fig. 6 illustrates the results of the 
inference process for an example, the formation of the 30S-mRNA complex.

\section{Computing performance}

Using HermiT 1.3.8 [29] within the Protégé editor, the consistency of BiPON can be computed in less than $5 \mathrm{~s}$. The automatic building of the class hierarchy (including the identification of inputs/outputs of aggregated processes and of key participants) and the inference of relationships between bioBiPON and modelBiPON takes $240 \mathrm{~min}(2.33 \mathrm{GHz}, 16 \mathrm{Go})$.

\section{Flexibility and genericity}

New aggregated processes can be easily defined by specifying (a) the elementary sub-processes to be aggregated using the has_subprocess sub-properties. The inputs/outputs of the aggregated process and the consumed molecules can then be inferred by automatic reasoning. In addition, participant element that have a key role in elementary or aggregated processes can be automatically determined. Altogether, combining DL syntax, SWRL rules and automatic reasoning make BiPON highly flexible and generic regarding the addition of new processes, participants, or models. Due to the flexibility of SWRL, new rules can be created and added easily. For test purposes, we provided a simple ontological model (toyBi$\mathrm{PON}$ ) that is representative of BiPON in Additional file 3. This model is schematically described in Additional file 4.

\section{BiPON content}

The set of BiPON statistical metrics and mapping is presented on Table 1. The ontology BiPON consists of 1746 classes (including 767 distinct individuals representing leaf-classes) and 30 object properties. Definitions of classes use 15,054 Axioms, including 4265 logical axioms, 8 SWRL rules for identification of the inputs/outputs of aggregated processes and 2 SWRL rules for alternative biological information feature (identification of key participants and consumed molecules).

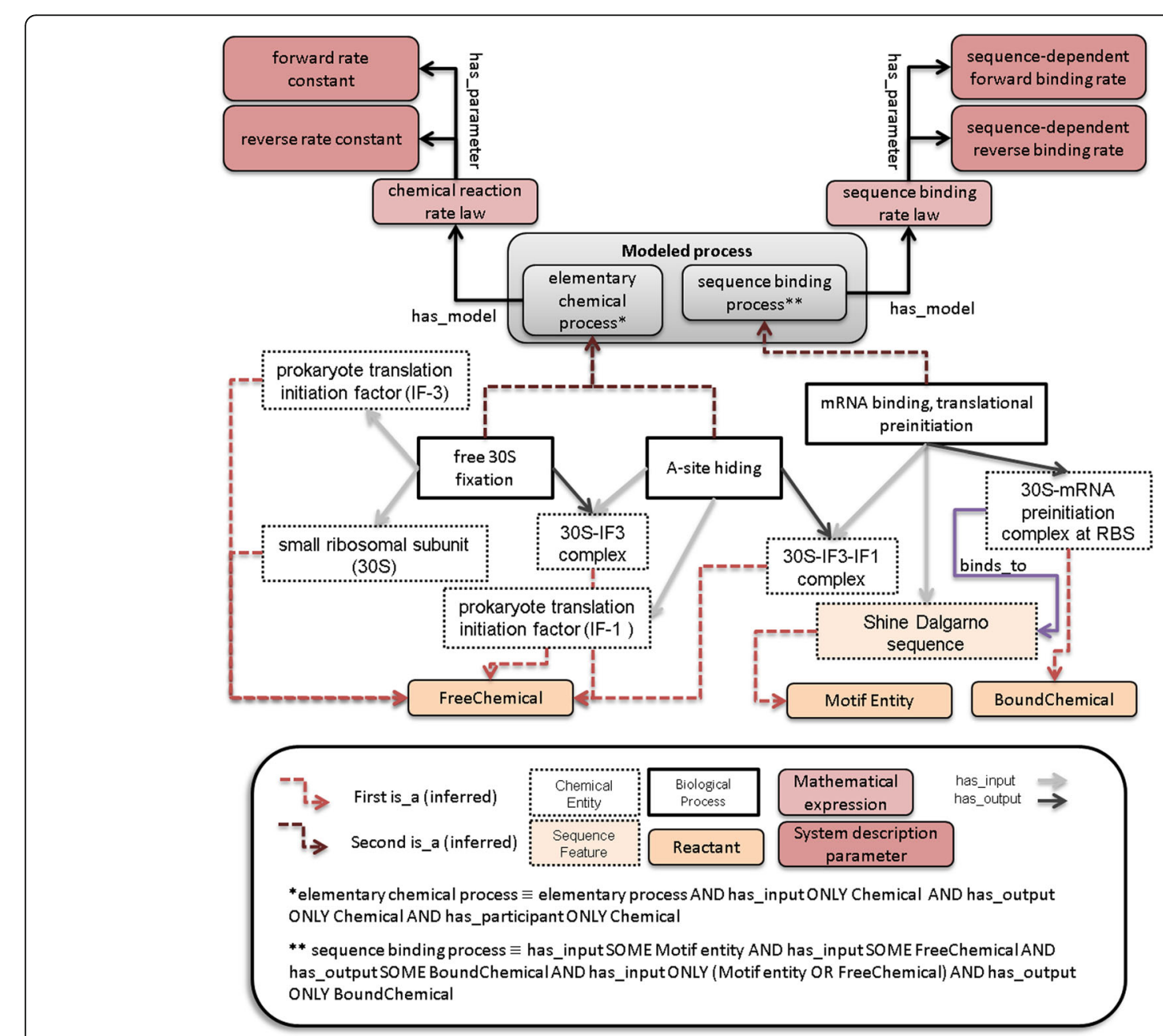

Fig. 6 Results of reasoning on bioBiPON and modelBiPON for elementary processes involved in the formation of the 30S-mRNA complex 
Table 1 Numbers and provenance of BiPON classes

\begin{tabular}{|c|c|c|c|c|c|c|c|}
\hline \multirow[b]{2}{*}{ Class name } & \multicolumn{2}{|l|}{ Numbers } & \multicolumn{5}{|c|}{ Provenance of classes (nb of classes) } \\
\hline & Class count & $\%$ of $\mathrm{BiPON}$ & GO & ChEBI & KEGG & SO & SBO \\
\hline BiPON & 1746 & $100,0 \%$ & 145 & 183 & 186 & 92 & 49 \\
\hline $\mathrm{L}$ modelBiPON & 194 & $11 \%$ & - & - & - & - & 49 \\
\hline L bioBiPON & 1582 & $91 \%$ & 145 & 183 & 186 & 92 & - \\
\hline L biological process & 551 & $32 \%$ & 123 & - & 34 & - & - \\
\hline $\mathrm{L}$ elementary process & 243 & $14 \%$ & 29 & - & 32 & - & - \\
\hline $\mathrm{L}$ aggregated process & 131 & $7 \%$ & 21 & - & - & - & - \\
\hline L Cellular component & 19 & $1 \%$ & 5 & 1 & - & 5 & - \\
\hline L Chemical entity & 754 & $43 \%$ & 17 & 183 & 152 & 41 & - \\
\hline $\mathrm{L}$ sequence feature & 234 & $13 \%$ & - & - & - & 51 & - \\
\hline input/output (i/o) ${ }^{\mathrm{a}}$ & 514 & $29 \%$ & 8 & 90 & 144 & 36 & - \\
\hline $\mathrm{L}$ i/o AND molecule & 34 & $2 \%$ & - & 34 & - & - & - \\
\hline Li/o AND macromolecule & 444 & $25 \%$ & 8 & 56 & 144 & 28 & - \\
\hline $\mathrm{L}$ i/o AND sequence feature & 36 & $2 \%$ & - & - & - & 8 & - \\
\hline
\end{tabular}

The proportions in the columns "Numbers" are computed with respect to the total class count (1746) of BiPON

a $\equiv$ Reactant

bioBiPON classes cover $91 \%$ of BiPON classes. One third of them are subclasses of Biological process, and two thirds are Chemical entity or Sequence feature subclasses. The difference in proportion is due to the fact that a Biological process is defined by different participants in input-output. $28 \%$ of the Biological process classes were imported from GO (123) and KEGG (34), while $45 \%$ of the participants were imported from GO (17), ChEBI (183), SO (92) and KEGG (152; Table 1). Classes imported from GO and SO were mainly used for hierarchy building, classes imported from ChEBI and especially KEGG were mostly used as leaf-classes. The remaining 976 non-imported classes (62\%) were manually designed. modelBiPON classes represent only $11 \%$ of BiPON classes due to their abstract representation. This low coverage of BiPON classes by modelBiPON classes is particularly interesting. Despite their apparent diversity, many biological processes can thus be represented by the same type of mathematical models. Most of modelBiPON classes are distributed between mathematical expression and system description parameters and 26\% of them were imported from SBO (49).

Biological processes include 243 elementary processes that are successively aggregated in 131 aggregated processes up to final aggregates. The elementary processes have 514 participants as input or output: 34 of this participants describes molecules, 444 gene products or molecular complex, 36 sequences. Altogether, molecules and sequence patterns represent only $14 \%$ of the inputs or outputs of biological processes, but they are involved in the definition of $63 \%$ of elementary processes. These proportions highlight the difference between basic molecules (e.g. water, ions or ATP) and sequence patterns (e.g. codons), which are often involved in many biological processes of gene expression, and macromolecules such as proteins, which are usually specific to a few biological processes.

After automatic reasoning, Modeled process classes include 213 elementary processes subclasses and 66 aggregated processes subclasses of bioBiPON. A large proportion among them (69\%) are Chemical process subclasses in modelBiPON and was automatically linked to the mathematical expression "chemical reaction rate law" and its parameters (see Fig. 5). The remaining 31\% correspond to other types of mathematical models, such as "sequence binding rate law" for Sequence binding process.

Currently, bioBiPON contains 77 biological processes subclasses that have no associated mathematical model and are therefore not included in modelBiPON. These classes correspond to intermediate processes that are not critical in a modeling perspective, but interesting for the biological knowledge description. However, in the future, if a mathematical model was built for them, it could be included in modelBiPON straightforwardly.

\section{Discussion}

In engineering science, the development of complex systems such as airplanes or nuclear power plants involves the development of specifications to ensure that the whole system will function in normal and/or degraded modes. As a prerequisite, such specifications will usually contain a catalog of sparse parts and an interaction map of the entire system. Each piece of the system must be described and characterized. To tackle the intrinsic complexity of managing thousands of entities, the design of the whole system is achieved by using a systemic 
approach: the whole system is broken down into subsystems of lower complexity, where each sub-system is well characterized and can be simulated. Adopting such a systemic representation was clearly the cornerstone of the development of complex engineering systems [11]. At the beginning of the twenty-first century, the field of systems biology was established based on exactly the same idea: that a cell is a complex system and that a systemic representation can help understand how the whole cell works [10]. Kitano's analysis in [10] appeared to be especially fruitful. Since 2012, the first developed whole-cell models were based on the systemic description of cells and showed a high capability of prediction $[1,2]$. This effectively demonstrates the relevance of the systemic approach to progress in the understanding of living organisms. Systems biology can thus greatly profit from systematically importing relevant concepts and know-how from engineering science into the biological field [10].

In this article, we introduced the ontology BiPON, which is intrinsically based on a systemic representation of cells $[1,6,7]$. In particular, BiPON focuses and clarifies the notion of biological processes by breaking down the cell into subsystems and by automatically relating them to mathematical models. Each subsystem is formally defined in terms of the cellular components it contains. Consequently, the function of each cellular component is conditioned to the subsystem to which it belongs. If a cellular component participates in multiple subsystems, it can also have different functions in the cell. In other words, the function of a cellular component now depends on the biological processes in which it is involved, and not only on its own chemical properties.

In describing biological knowledge, BiPON relies as much as possible on existing, well-established and commonly used bio-ontologies (GO, ChEBI, SO, SBO) in order to avoid the conception of unnecessary classes and, thus, to prevent redundancy. To ensure interoperability, we carefully stored IRI or Ids of each imported class and added new classes only when necessary. Approximately $72 \%$ of the Biological process classes in BiPON were created manually (see Table 1). Among the new Biological process classes, we created 175 elementary processes to further refine the biological description of bacterial cellular processes, thereby contributing to enrich the biological knowledge for prokaryotes. Finally, $78 \%$ of the Biological process classes in BiPON were linked to mathematical models by automatic reasoning. Altogether, combining the systemic description of the cell with an ontology enabled us to detect and fill gaps in the description of bacterial gene expression. BiPON is suited to help users to refer and share the same concepts regardless of their scientific background (biologist, mathematician, etc).
BiPON complements several ongoing efforts of the GO consortium, including GO-plus [31-33] and the Linked Expression using the Gene Ontology (GOCAM) formalism [32], which aim at promoting the comprehension, consistency and integration of biological knowledge within ontologies. GO-Plus provides relations between classes in GO-BP, GO-CC, GO-MF, metabolite classes in $\mathrm{ChEBI}$, and polymer classes in SO. Compared to GOplus, BiPON went a step further by integrating the $\mathrm{SO}$ sequence patterns involved in bacterial gene expression, and the mathematical models and related parameters of SBO. Specific sequence patterns had to be integrated because they are involved in $33 \%$ of elementary processes as participants. Beyond their content, GO-Plus, GOCAM and now BiPON provide promising formal frameworks to relate biological processes to their molecular functions. In [34, 35], the authors suggested that the formal relationship between GO-BP and GO-MF should be refined to improve the representation of biological knowledge. GO-Plus includes GO$\mathrm{BP}$ and GO-MF sub-ontologies [33] without the addition of new relations. Within the GOCAM project [32], the GO consortium has defined a new relation (affects) to link GOMF to GO-BP. Using BiPON, we expect to infer such links by automatic reasoning. For example, we automatically identified in BiPON the key participant of a biological process as the relevant Chemical entity that is necessary and unchanged during a Biological process. In the case of an enzymatic reaction, the key participant is the enzyme itself. Relating biological processes to molecular functions in BiPON could be achieved as follows: IF a chemical entity is a "key element" of a "process" and "has_function" a "molecular function", THEN this "molecular function" "affects" the "process" and all "aggregated process" of higher level (see Additional file 2 for the formal SWRL rule). We provided an example of such a reasoning in the toyBiPON ontology (see Additional file 3). Altogether, this illustrates the usefulness of bio-ontologies through the ability of inferring new relations by automatic reasoning.

Beyond that, ontologies are also widely used to organize data warehouses [36-38]. BiPON is currently used to drive the development of a data warehouse for the bacterial gene expression processes. BiPON supports the design of the relational model of the database that stores and connects biological knowledge, heterogeneous multi-omics data $[2,25,39,40]$, static data such as sequence patterns, and mathematical models [23, 24]. Highthroughput technologies already enable the acquisition of data at a large scale: transcriptome, proteome (including post-translational modifications), fluxome, interactome, metabolome, degradome, etc. [41]. In the future, new information could be obtained by combining omics data acquisition with statistical tools and computational algorithms for data analyses [42], model simulations, and accumulated biological knowledge. Typically, the combination of omics 
data and data analysis methods [43] may help to identify large sets of molecular compounds together with their biological functions, their interactions [44], and some of their kinetic properties, including for instance equilibrium constants or the half-lives of proteins or mRNAs. Using BiPON, any new omics data, new in silico predictions or new dynamical parameter sets (such as half-life of a protein, affinity constant, kinetic parameters, etc.) can be automatically anchored to systemic description of the cell and be linked to appropriate chemical entities or biological process of interest at any scale. BiPON and the data warehouse could serve as a point of entry into a shared resource of information that may be useful for biologists, computational biologists, statisticians and modelers.

BiPON currently contains a rather exhaustive description of the bacterial gene expression (including mechanisms of regulation), i.e. transcription, RNA processing and decay, ribosome biogenesis, tRNA aminoacylation and translation. However we are aware that BiPON is not yet complete and that new classes will have to be added in the future. In fact, the description methodology proposed in this article is highly flexible and generic. We expect that any new process, new participant, or other knowledge resource can be inserted in BiPON and be linked to mathematical models. BiPON is an ongoing project and future releases of BiPON will cover not only other bacterial processes such as DNA replication, cell wall synthesis or metabolism, but also cellular compartments (cytosol, membrane, periplasm, etc.). This will be a key step for extending BiPON to compartmentalized eukaryotic cells.

\section{Conclusions}

In this manuscript, we developed BiPON an ontology dedicated to the systemic representation of bacterial biological processes. This ontology is a proof of concept in several ways. It demonstrates that a large set of interlocked bacterial processes can be formally described, with an ontology, as input/output subsystems on different levels of granularity using a few set of properties and can automatically be linked through inference with their mathematical models and related parameters. The proposed methodology to build the systemic representation of bacterial processes is generic and could thus be easily implemented for other processes. BiPON links elementary entities such as single molecules or sequence patterns to biological processes and enables users to navigate from elementary to high-level processes and vice versa. Finally, combining instantiation and SWRL rules during automatic reasoning on BiPON enrich the knowledge by providing new properties with high flexibility. By interlacing biological knowledge with mathematical models, BiPON should open up promising perspectives for biologists, for data scientists, for computational, and system biologists and more largely for the emerging multi-disciplinary community of researchers studying whole-cell integration, modeling and simulation.

\section{Additional files}

Additional file 1: The list of the main references used to review the bacterial gene expression processes. (PDF $70 \mathrm{~kb}$ )

Additional file 2: Table of SWRL rules for the properties consumes, has_input, has_output, has_key_element, affects, precedes, before. (TIFF 90 kb)

Additional file 3: A small ontological model that illustrates the usage of BiPON. (OWL 199 kb)

Additional file 4: Graphical representation of the biological processes, chemical entities, and properties included in toyBiPON. (PNG 205 kb)

\section{Abbreviations \\ 30S: Prokaryote ribosomal small subunit; BioE: Biological entities; BiPON: Bacterial interlocked Process ONtology; cBioE: consumed BioE; ChEBI: Chemical Entities of Biological Interest; DAG: Directed Acyclic Graph; DL: Description Logic; fBioE: final BioE; GO: Gene Ontology; GO-BP: GO biological process; GO-CC: GO Cellular Component; GO-MF: GO Molecular Function; iBioE: initial BioE; IF3: Translation initiation factor; KEGG: Kyoto Encyclopedia of Genes and Genomes; mRNA: messenger RNA; ncBioE: new consumed BioE; OWL: Web Ontology Language; RF: Release Factor protein; RNA: RiboNucleic Acid; RO: Relation Ontology; SBO: System Biology Ontology; SO: Sequence Ontology; SWRL: Semantic Web Rule Language}

\section{Acknowledgements}

We thank the members of the IMSV project and Xavier Aimé for helpful discussions and Louise Deleger and Wolfram Liebermeister for critical comments on the manuscript. We are grateful to the INRA MIGALE bioinformatics platform (http://migale.jouy.inra.fr), especially to Sandra Dérozier for providing help and support.

\section{Funding}

This work has been funded by the French Lidex-IMSV of the University ParisSaclay.

\section{Availability of data and materials}

BiPON is distributed under the license Creative Commons Attribution 4.0 (CC-by; https://creativecommons.org/licenses/by/4.0/) and can be downloaded at http://maiage.jouy.inra.fr/?q=en/biosys/ontology and on BioPortal (http://bioportal.bioontology.org/ontologies/BIPON).

\section{Authors' contributions}

AG and VF designed the study. VH and AF developed the ontology with the help of SF, and inputs of VF, MD, VL and AG. VH and CF designed the hierarchical organization of the ontology and performed the automatic inference of classes and properties. VH, AG and CF drafted the manuscript. All authors critically revised the ontology and the manuscript. All authors read and approved the final manuscript.

\section{Ethics approval and consent to participate}

Not applicable.

\section{Consent for publication}

Not applicable.

\section{Competing interests}

The authors declare that they have no competing interests.

\section{Publisher's Note}

Springer Nature remains neutral with regard to jurisdictional claims in published maps and institutional affiliations. 


\section{Author details}

'Laboratoire de Recherche en Informatique (LRI), UMR 8623, CNRS, Université Paris-Sud/Université Paris-Saclay, Orsay, France. ${ }^{2}$ INRA, UR1404, MaIAGE, Université Paris-Saclay, Jouy-en-Josas, France.

\section{Received: 25 November 2016 Accepted: 10 November 2017}

Published online: 23 November 2017

\section{References}

1. Karr JR, Sanghvi JC, Macklin DN, Gutschow MV, Jacobs JM, et al. A wholecell computational model predicts phenotype from genotype. Cell. 2012; 150:389-401.

2. Goelzer A, Muntel J, Chubukov V, Jules M, et al. Quantitative prediction of genome-wide resource allocation in bacteria. Metab Eng. 2015;32:232-43.

3. Klipp E, Liebermeister W, Wierling C, Kowald A, Herwig R. Systems biology: a textbook. John Wiley and Sons. 2016;

4. Bodenreider O, Stevens R. Bio-ontologies: current trends and future directions. Brief Bioinform. 2006;7(3):256-74

5. Hoehndorf R, Schofield PN, Gkoutos GV. The role of ontologies in biological and biomedical research: a functional perspective. Brief Bioinform. 2015; 16(6):1069-80

6. Goelzer A, Fromion V, Scorletti G. Cell design in bacteria as a convex optimization problem. Automatica. 2011;47(6):1210-8.

7. Goelzer A, Fromion V. Bacterial growth rate reflects a bottleneck in resource allocation. Biochim Biophys Acta. 2011;1810(10):978-88.

8. Hartwell LH, Hopfield JJ, Leibler S, Murray AW. From molecular to modular cell biology. Nature. 1999;402:C47-52.

9. Goelzer A, Bekkal Brikci F, Martin-Verstraete I, Noirot P, Bessieres P, et al. Reconstruction and analysis of the genetic and metabolic regulatory networks of the central metabolism of Bacillus subtilis. BMC Syst Biol. 2008;2:20.

10. Kitano H. Foundations of systems biology. Cambridge. MIT press. 2001:1-36.

11. Blanchard BS, Fabrycky WJ, Fabrycky WJ. Systems engineering and analysis. Englewood Cliffs, NJ: Prentice Hall; 1990.

12. Hucka M, Finney A, Sauro $H$, Bolouri $H$, Doyle JC, Kitano $H$, Arkin AP, Bornstein B, Bray D, Cornish-Bowden A. Others. The systems biology markup language (SBML): a medium for representation and exchange of biochemical network models. Bioinformatics. 2003:19(4):524-31.

13. Hoehndorf R, Dumontier M, Gennari JH, Wimalaratne S, de Bono B, et al. Integrating systems biology models and biomedical ontologies. BMC Syst Biol. 2011;5:124.

14. Gene ontology consortium, et al. The gene ontology (GO) database and informatics resource. Nucleic Acids Res. 2004:32(1):D258-61.

15. Balakrishnan R, Harris MA, Huntley R, Van Auken K, Cherry JM. A guide to best practices for Gene Ontology (GO) manual annotation. Database 2013. 9:bat054.

16. Chibucos MC, Zweifel AE, Herrera JC, Meza W, Eslamfam S, Uetz P, et al. An ontology for microbial phenotypes. BMC Microbiol. 2014;14(1):294.

17. He Y, Liu Y, Zhao B. OGG: a biological ontology for representing genes and genomes in specific organisms. Proceedings in ICBO. 2014:13-20.

18. Eilbeck K, Lewis SE, Mungall CJ, Yandell M, Stein L, et al. The sequence ontology: a tool for the unification of genome annotations. Genome Biol. 2005:6(5):1.

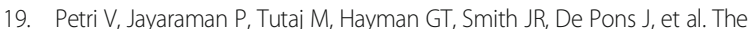
pathway ontology-updates and applications. J Biomed Semantics. 2014;5(1):7.

20. Hastings J, de Matos P, Dekker A, Ennis M, Harsha B, et al. The ChEB reference database and ontology for biologically relevant chemistry: enhancements for 2013. Nucleic Acids Res. 2013;41(1):D456-63.

21. Courtot M, Juty N, Knüpfer C, Waltemath D, Zhukova A, et al. Controlled vocabularies and semantics in systems biology. Mol sys. Biol. 2011:7(1):543.

22. Kanehisa M, Goto S, Sato Y, Furumichi M, Tanabe M. KEGG for integration and interpretation of large-scale molecular data sets. Nucleic Acids Res. 2011; gkr988.

23. King Z, Lu J, Dräger A, Miller P, Federowicz S, Lerman J, Ebrahim A, Palsson B. Lewis, N. E. BiGG models: a platform for integrating, standardizing and sharing genome-scale models. Nucleic Acids Res. 2016;44(D1):D515-22.

24. Juty N, Ali R, Glont M, Keating S, Rodriguez N, Swat M, Wimalaratne S, Hermjakob H, Le Novère N, Laibe C, Chelliah V. BioModels: content, features, functionality, and use. CPT Pharmacometrics Syst Pharmacol. 2015:4(2):55-68

25. Borkowski O, Goelzer A, Schaffer M, Calabre M, Mäder U, Aymerich S, et al. Translation elicits a growth rate-dependent, genome-wide, differential protein production in Bacillus Subtilis. Mol Syst Biol. 2016;12(5):870.
26. Fromion V, Leoncini $E$, Robert PA. Stochastic model of the production of multiple proteins in cells. SIAM J. Appl Math. 2015;75(6):2562-80.

27. Horridge M, Drummond N, Goodwin J, Rector AL, Stevens R, et al. The Manchester OWL syntax. OWLed. 2006:216.

28. Smith B, Ceusters W, Klagges B, Köhler J, Kumar A, et al. Relations in biomedical ontologies. Genome Biol. 2005;6(5):1.

29. Glimm B, Horrocks I, Motik B, Stoilos G, Wang Z. HermiT: an OWL 2 reasoner J Autom Reason. 2014;53(3):245-69.

30. Krisnadhi A, Maier F, Hitzler POWL. Rules. In: Reasoning web. Semantic Technologies for the web of data: Springer Berlin Heidelberg; 2011. p. 382-415.

31. Hill DP, Adams N, Bada M, Batchelor C, Berardini TZ, et al. Dovetailing biology and chemistry: integrating the gene ontology with the ChEBI chemical ontology. BMC Genomics. 2013;14(1):513.

32. The Gene Ontology Consortium. Expansion of the Gene Ontology knowledgebase and resources. Nucleic Acids Res. 2017; 4:45(D1):D331-D338.

33. Gene Ontology Consortium. Gene ontology consortium: going forward. Nucleic Acids Res 2015; 43(Database issue):D1049-D1056.

34. Hill DP, D'Eustachio P, Berardini TZ, Mungall CJ, Renedo N, Blake JA. Modeling biochemical pathway in the gene ontology. Database. 2016;1:2016.

35. Myhre S, Tveit H, Mollestad T, Laegreid A. Additional gene ontology structure for improved biological reasoning. Bioinformatics. 2006;15(22): 2020-7.

36. Pardillo J, Mazon JN. Using ontologies for the design of data warehouse. IJDMS. 2011:3:73-87.

37. Romero O, Abello A. Automating multidimensional design from ontologies. DOLAP '07. 2007:1-8.

38. Blake JA, Bult CJ. Beyond the data deluge: data integration and bioontologies. J Biomed Inform. 2006;39(3):314-20.

39. Nicolas P, Mäder U, Dervyn E, Rochat T, Leduc A, Pigeonneau N, et al. Condition-dependent transcriptome reveals high-level regulatory architecture in Bacillus Subtilis. Science. 2012:335(6072):1103-6.

40. Buescher JM, Liebermeister W, Jules M, Uhr M, Muntel J, Botella E, et al. Global network reorganization during dynamic adaptations of Bacillus Subtilis metabolism. Science. 2012;335(6072):1099-103.

41. Metzker ML. Sequencing technologies-the next generation. Nat Rev Genet. 2010;11:31-46

42. Nekrutenko A, Taylor J. Next-generation sequencing data interpretation: enhancing reproducibility and accessibility. Nat Rev Genet. 2012;13:667-72.

43. Henry VJ, Bandrowski AE, Pepin AS, Gonzalez BJ, Desfeux A. OMICtools: an informative directory for multi-omic data analysis. Database; 2014, bau069.

44. Zhang W, Li F, Nie L. Integrating multiple 'omics' analysis for microbial biology: application and methodologies. Microbiology. 2010;156(2):287-301.

\section{Submit your next manuscript to BioMed Central and we will help you at every step:}

- We accept pre-submission inquiries

- Our selector tool helps you to find the most relevant journal

- We provide round the clock customer support

- Convenient online submission

- Thorough peer review

- Inclusion in PubMed and all major indexing services

- Maximum visibility for your research

Submit your manuscript at www.biomedcentral.com/submit
) Biomed Central 